\title{
Molecular Foundry Workshop Draws Overflow Crowd to Berkeley Lab
}

Nanoscale science and technology is now one of the top research priorities in the United States. For evidence, one need look no further than the National Nanotechnology Initiative, emerging five-years ago under the aegis of the Clinton Administration and now continuing with gusto under the Presidency of George W. Bush. Spurred by a steady stream of exciting new research, funding under the initiative is growing rapidly. In fiscal year 2003, for example, the research portfolios planned for the National Science Foundation, the Department of Defense, and the Department of Energy (DOE) will swell to more than half a billion dollars for exploration of the world at the nanoscale.

With this background, it is no surprise that an overflow crowd or more than 350 registrants filled two auditoriums to hear about and contribute ideas for the new Molecular Foundry during a two-day workshop at the Lawrence Berkeley National Laboratory (Berkeley Lab). Scheduled to open for business at Berkeley Lab in early 2006, the Molecular Foundry is one of three Nanoscale Science Research Centers (NSRCs) put forward for funding by the DOE's Office of Basic Energy Sciences (BES).

The workshop began with the customary brief welcome by Berkeley Lab Director Charles Shank, who expressed his high hopes for the extraordinary science to emerge from the workshop participants and others who will be working at the Molecular Foundry. Next, Patricia Dehmer, DOE Associate Director of Science for Basic Energy Sciences, explained that the NSRC's were the centerpiece of DOE's contribution to the National Nanoscience Initiative, a position in line with the recommendations of the Interagency Working Group on Nanoscale Science, Engineering, and Technology (IWGN) of the National Science and Technology Council (NSTC), which has been coordinating the nanoscience initiative and urged that DOE make NSRCs part of its contribution to the NNI. Dehmer emphasized to those in attendance that they were expected to be active participants by expressing their views about the Foundry's proposed facilities and operation.

Daniel Chemla, who has triple duties as Director of Berkeley Lab's Materials Sciences Division (MSD) and the ALS and as leader of an active research program centered around semiconductor quantum wells, is generally considered to be the father of the Molecular Foundry concept. Appropriately, he laid the foundation for the workshop proceedings by presenting his view of the "Challenges and Opportunities in Nanoscience."

Combining the use of both "hard" and "soft" matter (e.g., inorganic physical and organic biological systems) in devices, the increasing importance of "complexity" (e.g., nonlinear and collective phenomena), the need to connect nanoscale objects to the macroscale world we live in, the necessity of moving from isolated nanodevices to complex functional assemblies, and the essential role of a fully equipped center where it can all be done under one roof while training the next generation of nanoscientists were themes of Chemla's presentation. 
In addition to Chemla's overview, there were three plenary lectures by experts exploring selected applications of nanoscale technology in their fields of expertise. J. Fraser Stoddard (University of California, Los Angeles) led off with a presentation bearing the intriguing title "The Mechanical Bond in Nanoarchitectonics." Among other accomplishments, Fraser's group garnered Science magazine's "Breakthrough of the Year" award in 2001 for building a functioning array of molecular-sized switches based in part on mechanical motion of interlocking organic molecules synthesized in the laboratory, a step toward a possible random-access memory (RAM).

Evelyn $\mathrm{Hu}$ (California Nanosystems Institute at the University of California, Santa Barbara) took the audience back to the "hard" matter side of the nanomaterials spectrum in her talk on "Crafting New Optical Materials from the Nanoscale." By embedding semconductor quantum dots (three-dimensional quantum wells) in a photonic bandgap matrix, her group was able, for example, to dramatically sharpen the broad spectrum collectively emitted by a random array of quantum dots to a narrow peak.

Gabor Somorjai (University of California, Berkeley, and Berkeley Lab) finished up with a description of his group's research on "Nanoparticles for Chemical Selectivity: Adsorption and Catalysis." Somorjai discussed ways to get the very high surface areas needed for catalysis and the use of selectively absorbing filters to protect against chemical attacks.

Attendees heard a series of detailed presentations about the Foundry, which will be headed by Paul Alivisatos (MSD and University of California, Berkeley, Department of Chemistry). Each of the DOE-sponsored NSRCs has a different flavor that reflects the strengths and resources available at the host laboratory. As described by Alivisatos, the Molecular Foundry, which organizationally will have its home in MSD, is to be organized around six core facilities, each directed by a senior scientist who already leads a strong research program with a nanoscience flavor.

The six facilities and their leaders are: Inorganic Nanomaterials (Alivisatos), Organic, Polymer/Biopolymer Synthesis (Jean Fréchet, MSD and UCB Department of Chemistry), Theory (Steve Louie, MSD and UCB Department of Physics), Nanofabrication (Jeff Bokor, MSD and UCB Department of Electrical Engineering and Computer Science), Biological Nanostructures (Carolyn Bertozzi, MSD and UCB Departments of Chemistry and Molecular and Cell Biology), and Imaging and Manipulation (Miquel Salmeron, MSD). Synergy and cross fertilization between facilities will be emphasized.

The Molecular Foundry will accommodate approximately 135 persons, including roughly 40 visitors, 30 students or postdoctoral fellows, and 65 scientific, technical, and administrative staff. Each facility will be supported by a mixture of staff scientists, technicians, postdoctoral associates, students, and administrative personnel. In addition, the staff will maintain active research programs of their own to ensure they remain at the forefronts of their fields. 
To be operated as a national facility, the Molecular Foundry will be available to scientists from universities, industry, and government laboratories whose research proposals have been peer reviewed by a Proposal Study Panel. The Molecular Foundry will welcome short-term and long-term visitors with various degrees of experience and collaborators. A combination of advanced equipment, collaborative staff, and breadth across disciplines will allow users to explore the frontiers of nanoscience. A typical scenario envisioned is one in which users will come to the Foundry with their ideas for nanostructures and use Foundry facilities to synthesize, characterize, and model materials that they can use to execute those ideas.

Those submitting successful proposals will come to a new six level building laboratory building with a total gross area of approximately 95,400 square feet that is to be sited between the National Center for Electron Microscopy (NCEM) and Building 66 (which houses much of Berkeley Lab's materials sciences research). The net usable area of approximately 53,000 square feet will include wet and dry laboratories, "specialty" rooms (controlled-temperature, low-vibration, and "clean" areas), laboratory support facilities and equipment rooms, conference and seminar rooms, and offices.

Included will be the equipment necessary to synthesize, manipulate, model, and characterize nanoscale materials and structures. Space will also be allocated to laboratories and offices for facility users. By also functioning as a "portal" to Berkeley Lab's established major user facilities, the Foundry will also leverage existing nanoscience research capabilities at the ALS, the NCEM, and the National Energy Research Scientific Computing Center (NERSC).

In addition to discussions after each presentation and the usual informal interactions in the hallways, parallel breakout sessions provided the main opportunities for audience participation. There were two sets of breakouts, each of which included one group for each of the six Molecular Foundry facilities. To set the stage for discussion, a facilitator made a brief presentation on a subject relevant to the specific facility. The first day ended with a reception and a barbecue-style buffet at the Berkeley Lab cafeteria, while the Molecular Foundry Scientific Advisory Board, which provides oversight of the facility for Berkeley Lab, held its inaugural meeting.

The workshop's second day featured overviews of the other two NSRCs selected for DOE funding - the Center for Nanophase Materials Sciences at Oak Ridge National Laboratory, to be operational in October 2005, and the Center for Integrated Nanotechnologies in Albuquerque, NM, under the joint sponsorship of Sandia National Laboratories and Los Alamos National Laboratory - by Ward Plummer (University of Tennessee) and Don Parkin (Los Alamos National Laboratory). (Proposals are also under consideration at DOE from Argonne National Laboratory and Brookhaven National Laboratory.) Summaries of nanoscience-related activities at NCEM (Uli Dahmen), the ALS (Neville Smith), and NERSC (Horst Simon) ended the early-morning activities.

Scheduled for a lunchtime ending, the workshop concluded with a lengthy discussion session, a report from Alivisatos summarizing the numerous issues raised by participants, 
and closing comments from DOE's Dehmer and Iran Thomas, DOE Deputy Associate Director for Science. Reflecting a highly enthusiastic response to plans for the Molecular Foundry, Alivisatos reported that the single-most-asked question was "Why aren't you starting sooner?" Dehmer noted that the same question arose at workshops held for the other nanoscience centers and affirmed DOE's commitment to work with facility directors to move as quickly as possible.

Art Robinson

Advanced Light Source

Berkeley Lab 


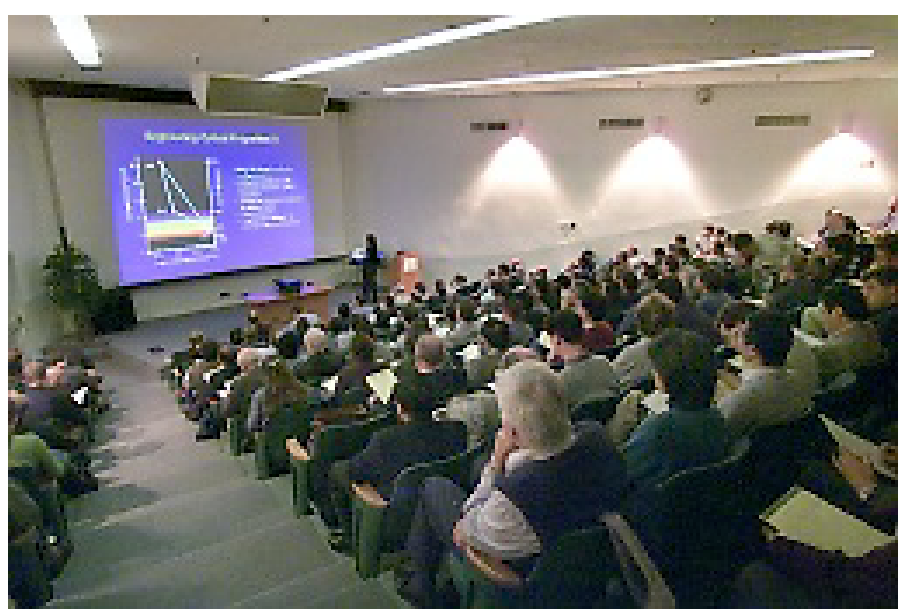

Figure 1. [XBD200204-00169-28]

An overflow crowd of more than 350 filled Berkeley Lab's main auditorium and much of a satellite auditorium equipped with video and audio feeds. Evelyn Hu (University of California, Santa Barbara) was one of the plenary session speakers who summarized opportunities for nanostructures in their research areas. 


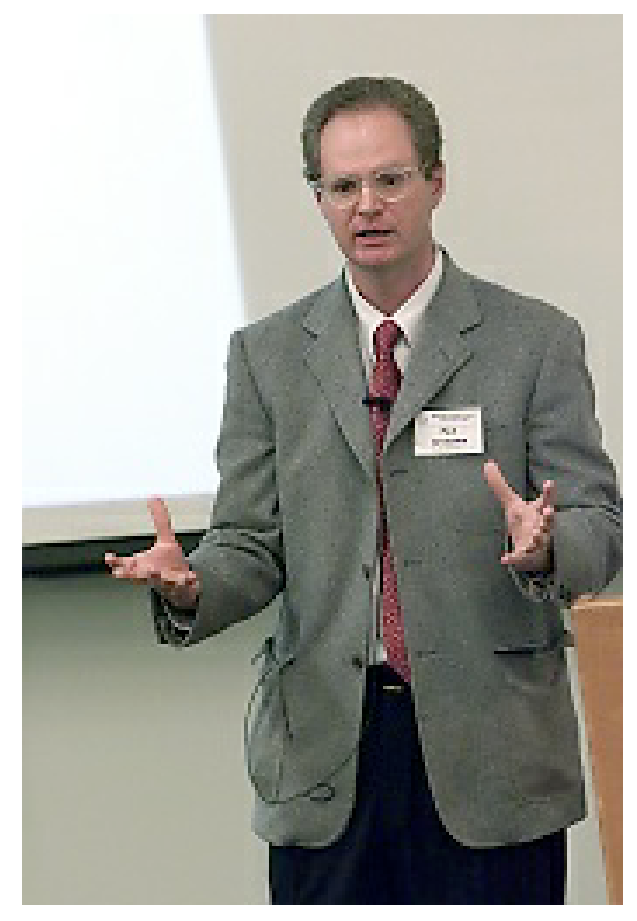

Figure 2. [XBD200204-00169-18]

Paul Alivisatos (University of California, Berkeley, and Berkeley Lab), Director of the Moleular Foundry, introduces workshop participants to an overall view of the facility concept and some specifics about its plans. 


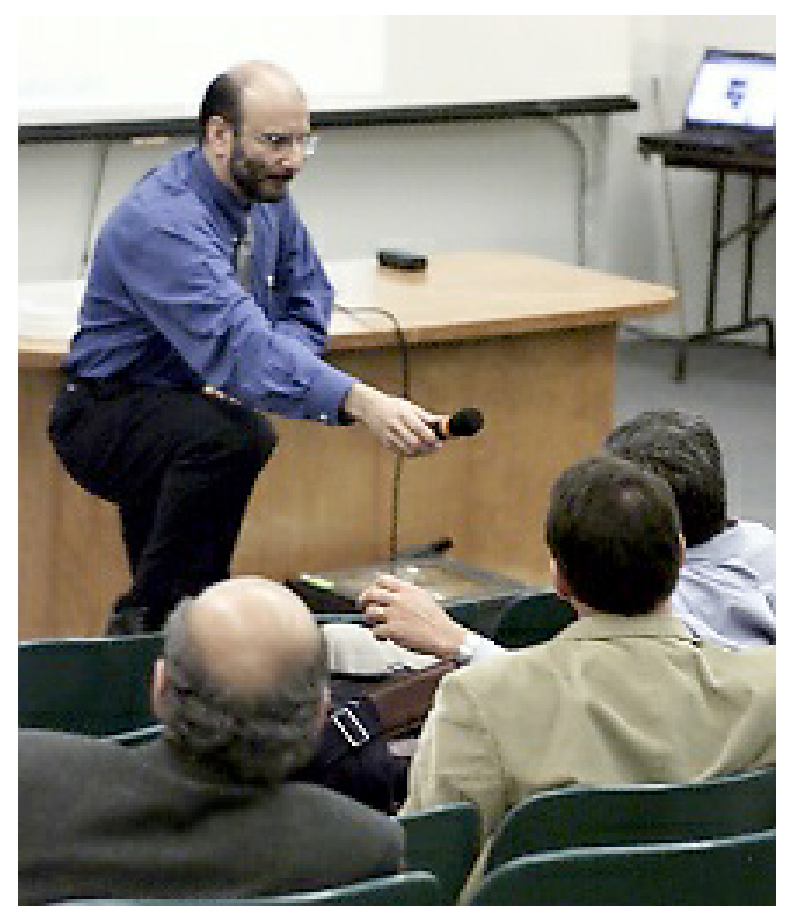

Figure 3 [XBD200204-00170-12]

Participation by those attending was a key component of the workshop proceedings, as in this breakout session on nanofabrication with Jeff Bokor (University of California, Berkeley, and Berkeley Lab) as the facilitator. 